\title{
Early top-pair cross-section measurements at ATLAS
}

\section{Urbaniec* on behalf of the ATLAS Collaboration}

Columbia University, New York, NY 10027, USA

E-mail: dustin.henry.urbaniec@cern.ch

\begin{abstract}
Top-quark pairs are expected to be produced copiously at the LHC, even at the lower beam energies and luminosities expected in the first year of running. Establishing the top-pair signal and measuring the production cross-section will be important benchmarks for ATLAS, which will also help understand the detector performance for events with high $p_{\mathrm{T}}$ leptons, high jet multiplicity, missing transverse energy and $b$-jets. The prospects for early measurements with top-quark pairs at a centre of mass energy of $10 \mathrm{TeV}$ will be shown, with a particular emphasis on the understanding of backgrounds through data-driven methods.
\end{abstract}

XXth Hadron Collider Physics Symposium

November 16 - 20, 2009

Evian, France

*Speaker. 


\section{Introduction}

After QCD jets, $W$, and $Z$ bosons, the production of top-quarks will be the dominant process at the LHC. The measurment of the cross-section of top-quark pair production is important for many reasons. It will provide an important test of the Standard Model as theoretical predictions are now at the level of around $10 \%$. An abundant $t \bar{t}$ sample will also serve as a useful calibration tool for reconstructed objects such as jets and missing $E_{\mathrm{T}}$. In addition, $t \bar{t}$ events will be a significant background for many new physics searches. Thus, a well understood $t \bar{t}$ cross-section measurement is essential at the LHC. In the Standard Model, top-quarks decay almost exclusively into a $W$ boson and a $b$-quark. Top-pair events are characterized by the number of $W$ bosons that decay leptonically. Considered in section 2 is the dilepton channel [1], where both $W$ bosons decay leptonically. In section 3, the single lepton channel [2] is discussed, where one $W$ decays leptonically and the other hadronically. The studies presented here are based on simulations with $10 \mathrm{TeV} p p$ collisions.

\section{Dilepton Channel}

Though the dilepton $t \bar{t}$ branching ratios are small, the presence of two leptons provides a very clean signature. Dilepton $t \bar{t}$ events are selected by requiring exactly 2 tight opposite sign leptons (electrons or muons) with $p_{\mathrm{T}}>20 \mathrm{GeV}$ and $|\eta|<2.5, \not_{\mathrm{T}}>35 \mathrm{GeV}$ for $e e$ and $\mu \mu$ events or $\not_{\mathrm{T}}>20 \mathrm{GeV}$ for $e \mu$ events, and at least 2 jets with $p_{\mathrm{T}}>20 \mathrm{GeV}$ and $|\eta|<2.5$. For the $e e$ and $\mu \mu$ channels, events with a dilepton invariant mass within $5 \mathrm{GeV}$ of the $\mathrm{Z}$ mass $(91 \mathrm{GeV})$ are vetoed in order to further suppress the Drell-Yan background.

Figure 1 shows the jet multiplicity distributions for the different contributions after all cuts except on the number of jets. Due to difficulties in modeling the background distributions in the tail regions, methods for background estimation using data driven techniques have been developed. The largest contributions are expected to be the Drell-Yan background as well as fakes coming from QCD and $W+$ jets.

As outlined above, two main selections are used to suppress the Drell-Yan events: one on $Z_{\mathrm{T}}$ and one on the invariant mass of the two same flavour leptons. The Drell-Yan background is estimated by scaling the Monte Carlo prediction in the tail regions of each of these distributions individually (where Drell-Yan still dominates) to match the observed number of events in the corresponding tail regions of data and then extrapolating to the signal region.

To estimate the background coming from fakes, we first measure the efficiency $\varepsilon$ (using a tag and probe method on $Z \rightarrow l l$ events) and the fake rate $f$ (using data samples dominated by fakes) of our lepton selections. A loose and tight lepton selection is defined and three different types of reconstructed events are considered: events with two tight leptons and events where the highest $p_{\mathrm{T}}$ lepton is tight (loose) and the other loose (tight). At the truth level, events again come in three types: events with two real leptons and events where the highest $p_{\mathrm{T}}$ lepton is real (fake) and the other fake (real). The truth level events are related to the reconstruction level events through a matrix equation using the measured fake rates and efficiencies. With this equation, an estimate for the number of events coming from fakes can be determined. 

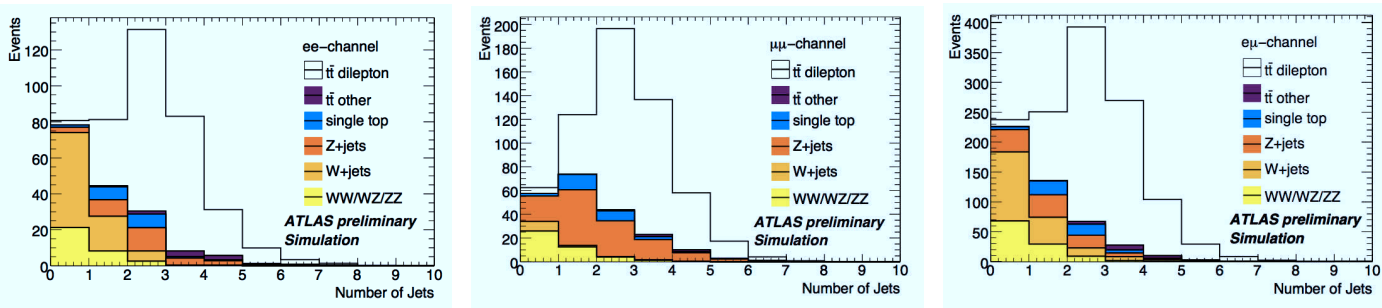

Figure 1: Jet multiplicity distributions for the $e e$-channel (left), $\mu \mu$-channel (centre), and $e \mu$-channel (right)

The cross-section is extracted by maximizing the following likelihood function:

$$
L\left(\sigma_{\text {sig }}, \mathscr{L}, \alpha_{j}\right)=\prod_{i \in \text { bins }}\left[\operatorname{Pois}\left(N_{i}^{\mathrm{obs}} \mid N_{i, \text { tot }}^{\mathrm{exp}}\right) \operatorname{Gaus}\left(\tilde{\mathscr{L}} \mid \mathscr{L}, \sigma_{\mathscr{L}}\right) \prod_{j} \operatorname{Gaus}\left(\tilde{\alpha}_{j}=0 \mid \alpha_{j}, \Delta_{\alpha_{j}}=1\right)\right]
$$

where $N_{i}^{\mathrm{obs}}$ is the number of observed events in the $i^{\text {th }}$ jet multiplicity bin, $N_{i, \text { tot }}^{\mathrm{exp}}$ is the number of expected events in that bin, $\mathscr{L}$ is the luminosity (with $\tilde{\mathscr{L}}$ being the nominal value and $\sigma_{\mathscr{L}}$ the uncertaintiy), and the $\alpha_{j}$ represent the various systematic uncertainties grouped such that the corresponding variations in the efficiencies $\varepsilon$ are expected to be uncorrelated. The final systematic uncertainty is estimated from the likelihood profile defined as $\lambda\left(\sigma_{\text {sig }}\right)=\frac{L\left(\sigma_{\text {sig }}, \hat{\mathscr{L}}_{,}, \hat{\alpha}_{j}\right)}{L\left(\hat{\sigma}_{\text {sig }}, \hat{\mathscr{L}}_{, j}\right)}$, where $\hat{\hat{\mathscr{L}}}$ and $\hat{\hat{\alpha}}_{j}$ are found from maximizing the likelihood while holding $\sigma_{\text {sig }}$ fixed. The distribution $-2 \log \lambda\left(\sigma_{\text {sig }}^{\text {true }}\right)$ is used along with Wilk's theorem to establish a confidence interval. The expected number of signal events after all selections, the signal-to-background ratio, and the statistical and systematic uncertainties with $200 \mathrm{pb}^{-1}$ at $10 \mathrm{TeV}$ for each channel are shown in table 1.

\section{Single Lepton Analysis}

The single lepton channel has the benefit of higher statistics due to higher branching fractions than the dilepton case (about $15 \%$ for each flavour). Events are selected by requiring exactly 1 tight lepton (electron or muon) with $p_{\mathrm{T}}>20 \mathrm{GeV}$ and $|\eta|<2.5, E_{\mathrm{T}}>20 \mathrm{GeV}$, and at least 4 jets with $|\eta|<2.5$ and $p_{\mathrm{T}}>20 \mathrm{GeV}, 3$ of which with $p_{\mathrm{T}}>40 \mathrm{GeV}$. A hadronic top-quark candidate is reconstructed by finding the 3 jet combination whose total $p_{\mathrm{T}}$ is the greatest among all 3 jet combinations. At this point, an optional $M_{W}$-cut can be applied by requiring that at least one of the three 2 jet combinations is within $10 \mathrm{GeV}$ of the $W$ mass.

It is possible that an accurate understanding of the missing $E_{\mathrm{T}}$ measurement will require substantial commissioning. One variation in the selections is to perform the measurement without a missing $E_{\mathrm{T}}$ cut in favor of a cut on HT2 (scalar sum of the $p_{\mathrm{T}}$ of the lepton and the $2^{\text {nd }}, 3^{\text {rd }}$, and $4^{\text {th }}$ jet). This is motivated by the fact that in QCD, the energy tends to be dominated by the two leading jets, in contrast to $t \bar{t}$ events which share the energy more equally. The lepton $p_{\mathrm{T}}$ and $\eta$ cuts are tightened in this variation of the analysis.

The easiest way to determine the cross-section is to use a cut-and-count method using the equation $\sigma=\frac{N_{\mathrm{sig}}}{\mathscr{L} \times \varepsilon}=\frac{N_{\mathrm{obs}}-N_{\mathrm{bkg}}}{\mathscr{L} \times \varepsilon}$, where $\mathscr{L}$ is the luminosity and $\varepsilon$ is the signal efficiency. One of the most important backgrounds in this analysis is $W$ boson production in association with jets. The Monte Carlo predictions for the fraction of $W$ events which are produced with 4 or more jets 

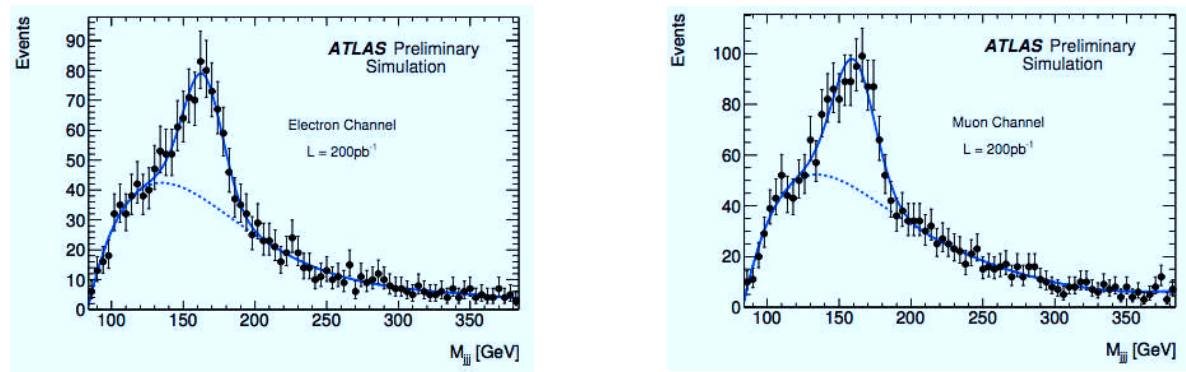

Figure 2: Fit to the top-quark candidate invariant mass distribution $\left(m_{j j j}\right)$ for the $e+$ jets channel (left) and the $\mu+$ jets channel (right)

have a large uncertainty. Since the $W$ to $Z$ ratio uncertainty is smaller, we can estimate the $W$ contribution to the signal region by measuring the ratio of $W$ to $Z$ events in a control region in data and extrapolating to the signal region. The expected number of signal events and the expected uncertainties using the cut-and-count method for $200 \mathrm{pb}^{-1}$ at $10 \mathrm{TeV}$ are shown in table 1 .

Another way to extract the cross-section is to do a fit to the 3 jet invariant mass to determine the signal size. After the $M_{W}$-cut, a binned maximum likelihood fit is performed with a Gaussian for the 3 jet invariant mass and a 6th order Chebyshev polynomial for the background. The integral of the fitted Gaussian is then used as an estimator for the number of correctly reconstructed $t \bar{t}$ events. The fits for each channel are shown in figure 2 .

\section{Summary}

Some of the methods used to measure the top-pair cross-section with early data at ATLAS have been summarized. For the dilepton channels, a simple set of selections have been studied and a likelihood method to extract the cross-section has been developed. For the single lepton channels, again a simple set of selections have been defined along with several variations. Both cut-andcount and fit methods for extracting the cross-section have been studied. Expected sensitivities at $\sqrt{s}=10 \mathrm{TeV}$ for $200 \mathrm{pb}^{-1}$ for all channels are summarized in table 1.

\begin{tabular}{|l||c|c||c|c|c|}
\hline Channel & $\mathbf{S}$ & $\mathbf{S} / \mathbf{B}$ & $\Delta \sigma($ stat $) / \sigma$ & $\Delta \sigma($ syst $) / \sigma$ & $\Delta \sigma($ lumi $) / \sigma$ \\
\hline$e e$ & 209 & 3.9 & $+7.8 /-7.5$ & $+13.9 /-12.7$ & $+26.3 /-17.3$ \\
\hline$\mu \mu$ & 327 & 3.8 & $+6.2 /-6.0$ & $+10.2 /-8.9$ & $+26.2 /-17.4$ \\
\hline$e \mu$ & 683 & 5.6 & $+4.1 /-4.0$ & $+10.2 /-9.4$ & $+26.2 /-17.4$ \\
\hline \hline$e+$ jets & 1286 & 2.1 & \pm 3.4 & $+14.4 /-15.2$ & \pm 22.3 \\
\hline$\mu+$ jets & 1584 & 2.0 & \pm 3.1 & $+11.9 /-14.7$ & \pm 22.2 \\
\hline
\end{tabular}

Table 1: Table showing expected number of signal events, signal-to-background ratio, and uncertainties with $200 \mathrm{pb}^{-1}$ of $10 \mathrm{TeV}$ data. The single lepton channels show results using the cut-and-count method.

\section{References}

[1] The ATLAS Collaboration, Prospects for Measuring Top Pair Production in the Dilepton Channel with Early ATLAS Data at $\sqrt{s}=10 \mathrm{TeV}$, ATL-PHYS-PUB-2009-086

[2] The ATLAS Collaboration, Prospects for the Top Pair Production Cross-section at $\sqrt{s}=10 \mathrm{TeV}$ in the Single Lepton Channel in ATLAS, ATL-PHYS-PUB-2009-087 